\title{
Rethinking Educational Transitioning From Grade 3 to Grade 4
}

\author{
Blandina Manditereza \\ Educare Department, Motheo Tvet College, Bloemfontein: South Africa
}

\begin{abstract}
In South Africa, Grade 3 learners are taught by a class teacher but once they move to Grade 4 the learners are now taught by subject teachers, meaning the learners will suddenly have maybe 4 to 5 teachers and must adapt to various pedagogical approaches. The challenge now is Grade 3 learner performance is usually satisfactory but once in Grade 4 the learners drop in academic achievement. The study sought to investigate the foundational problems that have plagued Grade 3-4 learners during transitioning. This study is significant in that it gives suggestive solutions which may address transitioning challenges. The Interpretive worldviews and the Case study approach were used alongside qualitative data collection methods. In the study 5 Ex Model C Schools were conveniently selected and the participants constituted 10 purposefully selected, Grade 3 teachers and 10 Grade 4 teachers as well. The following research instruments were used, lesson observations, interviews, and document analysis. Bronfenbrenner's Ecological theory was thus used as the theoretical lens which explored the pedagogical relationship between the teacher and the learner. Findings in the study indicated that when Grade 3 learners transition to Grade 4 they fail to adapt to environmental demands and the teacher pedagogy creates a learning gap to the once high performers, possibly because of the barrier created by the language of learning.
\end{abstract}

Keywords: context, culture, curriculum, ecological, transitioning. 